\title{
A Composite State Convergence Scheme for Multilateral Teleoperation Systems
}

\author{
Muhammad Usman ASAD ${ }^{1}$, Jason GU ${ }^{1}$, Umar FAROOQ ${ }^{1}$, Valentina E. BALAS ${ }^{2 *}$, \\ Zheng CHEN ${ }^{3}$, Chunqi CHANG ${ }^{4}$, Athar HANIF ${ }^{5}$ \\ ${ }^{1}$ Department of Electrical and Computer Engineering, Dalhousie University, Halifax, B3H 4R2, NS, Canada \\ mh549096@dal.ca, Jason.gu@dal.ca, umar.farooq@dal.ca \\ ${ }^{2}$ Automatics \& Applied Informatics, Aurel Vlaicu University of Arad, Arad, 310130, Romania \\ valentina.balas@uav.ro,balas@drbalas.ro (*Corresponding author) \\ ${ }^{3}$ Ocean College, Zhejiang University, Hangzhou, 310027, Zhejiang, China \\ zheng_chen@zju.edu.cn \\ ${ }^{4}$ School of Biomedical Engineering, Health Science Center, Shenzhen University, Shenzhen, 518060, China \\ cqchang@szu.edu.cn \\ ${ }^{5}$ Center for Automotive Research, College of Engineering, The Ohio State University, Columbus, Ohio, 43213 \\ hanif.6@osu.edu
}

\begin{abstract}
Composite state convergence scheme is a reduced-complexity version of the state convergence controller for teleoperation system. It employs a smaller number of control gains and communication channels used to synchronize the motion of a single master-slave system in a desired dynamic way. The present study aims at generalizing the composite state convergence scheme so that $l$-slave systems can follow the weighted motion of $k$-master systems. To achieve this, at first, composite variables of all master and slave systems are transmitted across the communication channel along with operators' forces and a set of $k+l+2 k l$ control gains is defined. In the second stage, the design procedure of the existing composite state convergence scheme is extended for multiple systems and the control gains are determined through the solution of coupled equations. Finally, to validate the findings, simulations and semi-real time experiments are performed in MATLAB/Simulink/QUARC environment by considering different configurations of teleoperation systems.
\end{abstract}

Keywords: State convergence, Composite variables, Linear systems, Teleoperation, Time delay, MATLAB/Simulink.

\section{Introduction}

Teleoperation refers to the execution of a task in a remote environment using master and slave robotic systems. These constituent robotic systems exchange various signals over the communication channel to ensure the smooth execution of the task. The communication channel while being the bridge between the master and slave systems also plays a negative role as it injects time delay in the signals which can lead to the instability of the teleoperation system (Ferre et al., 2007). To overcome the destabilizing effect of time delays in teleoperation systems, several control algorithms have been proposed in the specialized literature (Hokayem \& Spong, 2006; Arcara \& Melchiorri, 2002; Muradore \& Fiorini, 2016). A remarkable work in teleoperation systems dates back to 1980's when Anderson and Spong proposed a time delay compensation algorithm by using transmission line theory (Anderson \& Spong, 1989). This idea laid the foundation of wave variables (Niemeyer $\&$ Slotine, 1991) which, since then, has remained a popular choice for the design of teleoperation systems. The wave variable approach has also been revisited by many researchers with an aim to reduce its conservatism (D'Amore \&
Akin, 2017). Some researchers have proposed hybrid approaches based on wave variables and intelligent techniques to simultaneously deal with the time delays and the model uncertainties (Yang et al., 2017). Other control algorithms investigated by the researchers include time domain passivity control (Ryu et al., 2004), sliding mode control (Hace \& Franc, 2013), stability analysis via generalized inequalities (Datta et al., 2019), adaptive control (Chan et al., 2014), H- $\infty$ control (Yan \& Salcudean, 1996), fuzzy logic control (Yang et al., 2015), compensating time delays and stability analysis via digital controller based on polynomial approach (Del Muro Cuéllar et al., 2020; Farooq et al., 2016; Boukhnifer et al., 2012), disturbance observer-based control (Suzuki \& Ohnishi, 2013; Zhao et al., 2019; Nozaki et al., 2014), model predictive control (Uddin \& Ryu, 2016) and state convergence (Azorin et al., 2004). Amongst these control algorithms, state convergence is easy to design and implement but it can tolerate only small time delays. The easiness stems from the requirement of solving only $3 n+1$ design equation to determine control gains for a single pair of master and slave systems 
modelled on state space. A less complex version of state convergence controller is proposed and it is termed as composite state convergence controller (Asad et al., 2019a). The reduction in complexity is achieved through the transmission of two composite variables in place of $2 n$ (master and slave) states while force transmission remains the same in both cases. In total, only three variables are transmitted and only four control gains need to be determined in case of composite state convergence scheme. To deal with nonlinear systems, composite state convergence scheme is combined with feedback linearization technology (Asad et al., 2019b).

The use of multiple master and slave devices to perform a certain task is also gaining attention as this can improve the efficacy, loading capacity and handling capability of the teleoperation system. To this end, existing bilateral control algorithms are being extended to accomplish the goals ( $\mathrm{Li}$ et al., 2013; Chen et al., 2016; Li et al., 2016; Farooq et al., 2017; Farooq et al., 2018). To enhance the capability of composite state convergence, this paper proposes a generalized version of the said scheme where arbitrary number of master and slave devices can be added. The proposed extension ensures the synchronization of $l$-slaves with the references set by $k$-master systems through a proper selection of control gains which should satisfy $k+l+2 k l$ design conditions. These design equalities are the result of enforcing desired responses on the composite-master and composite-error systems with the latter being considered as autonomous systems. To validate the proposed scheme, MATLAB simulations are performed on different arrangements of teleoperation systems. Simulation results and semi-real time experiments reveal that the proposed scheme can successfully establish the synchronization as slave systems are able to track the weighted references of the master systems. This paper thus has the following contributions:

1. A generalization of the composite state convergence scheme is reported which enables $l$-slave systems to track $k$-master systems;

2. The number of communication channels is educed as compared to the extended state convergence architecture. In addition, the number of control gains is also reduced as compared to the extended state convergence architecture;
3. It is shown that synchronization of $l$ - composite-slave systems with $k$ - compositemaster systems guarantees the synchronization of original $l$-slave systems with the original $k$-master systems under the proposed systematic design procedure. In addition, stability of the proposed scheme is verified through Lyapunov analysis;

4. The proposed scheme is validated through simulations and semi-real time experiments in MATLAB, Simulink and QUARC environment.

The rest of the paper is organized as follows: Composite state convergence scheme is briefly reviewed in Section 2. The proposed generalization is presented in Section 3 while MATLAB simulations and experimental results are included in Sections 4 and 5, respectively. Conclusions are given in Section 6 and the stability analysis is provided in Appendix ' $A$ '.

\section{Review of Composite State Convergence Scheme}

Composite state convergence scheme establishes bilateral communication (position and force tracking) between a pair of master and slave systems by utilizing lesser control gains and transmitting fewer variables when compared to the original state convergence technique. In this scheme, the operator exerts a force on the master robotic system $\left(F_{m}\right)$. The resulting position and velocity signals of the master robotic system are fused to form a master's composite variable $\left(s_{m}\right)$. The operators force and the master's composite variable are then transmitted over the communication channel to the slave robotic system. Here, communication channel presents a constant time delay $(T)$ to the incoming signals. Thus, slave robotic system receives a delayed copy of the operator's force and master composite variable which influences the slave robotic system to interact with the environment. During this interaction, position and velocity signals of the slave robotic system are combined to form a slave's composite variable $\left(s_{s}\right)$, which is transmitted over the communication channel to the operator's side. In response, the operator adjusts the movement of the master robotic system to maintain a transparent teleoperation. The control gains which define composite state convergence scheme include operator's force scaling gain $\left(G_{2}\right)$, stabilizing gain for the composite-master system $\left(k_{m}\right)$, stabilizing gain for the composite-slave 
system $\left(k_{s}\right)$, and cross-coupling gains $\left(r_{m}, r_{s}\right)$ to scale the delayed composite variables. Among various parameters, $F_{m}, s_{m}, s_{s}, r_{m}, T$ are known scalars while unknown scalars $G_{2}, k_{m}, k_{s}, r_{s}$ are found through the composite-version of the state convergence methodology (Asad et. al., 2019). This scheme fixes the dynamics of the compositemaster and composite-error systems after allowing the composite-error to evolve as an autonomous system. Figure 1 shows the composite state convergence scheme.

Remark 1: Although composite state convergence scheme offers lower complexity (three communication channels and four design variables) as compared to its standard counterpart $(2 n+1$ communication channel and $3 n+1$ design variables), a generalization is desired in order for the scheme to accommodate any number of master and slave systems involved in the joint task. This has been a motivation to investigate the possibility of extending composite state convergence scheme for multiple systems.

\section{Proposed Composite State Convergence Scheme}

The proposed extension enables composite state convergence scheme to synchronize $l$-slave systems with the reference motions generated by $k$-master systems. The objective is to allow $j^{\text {th }}$ slave system to track combined motions of $k$-master systems as:

$x_{s}^{j}-\sum_{i=1}^{k} \alpha_{s j}^{i} x_{m}^{i} \rightarrow 0, t \rightarrow \infty$

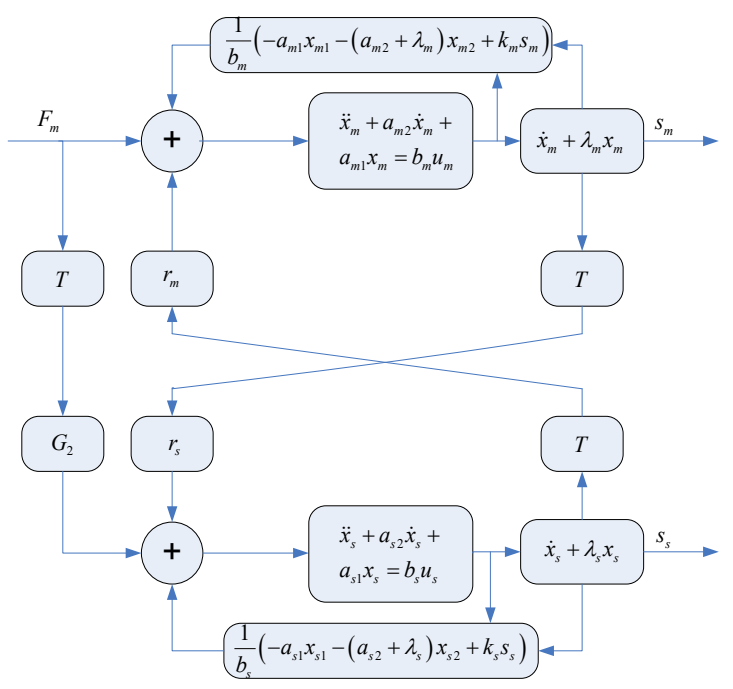

Figure 1. Composite state convergence scheme (Asad et al., 2019a) where $x$ denotes the states while $\alpha_{s j}^{i}$ are defined as authority factors for the master systems affecting $j^{\text {th }}$ slave system such that:

$$
\sum_{i=1}^{k} \alpha_{s j}^{i}=1
$$

\subsection{Communication Structure}

To achieve this objective, communication is first established by transmitting composite variables from all the master systems $\left(s_{m}^{j}, j=1,2, \ldots, k\right)$ to all the slave systems as well as from all the slave systems $\left(s_{m}^{j}, j=1,2, \ldots, k\right)$ to all the master systems over the communication channel, which offers constant time delays to the incoming signals. Here, $T_{m i}^{j}$ is the time delay from the $j^{\text {th }}$ slave system to the $i^{\text {th }}$ master system while $T_{s i}^{j}$ is the time delay from the $j^{\text {th }}$ master system to the $i^{\text {th }}$ slave system. Thus, $j^{\text {th }}$ slave system will receive delayed copies of composite variables of all $k$-master systems $\left(s_{m j d}^{i}=s_{m}^{i}\left(t-T_{s j}^{i}\right), i=1,2, \ldots, k\right)$ while $j^{\text {th }}$ master system will receive delayed copies of composite variables of all $l$-slave systems $\left(s_{s j d}^{i}=s_{s}^{i}\left(t-T_{m j}^{i}\right), i=1,2, \ldots, l\right)$. In addition, all the operators' forces $\left(F_{m}^{j}, j=1,2, \ldots, k\right)$ are also transmitted to the slave systems over the communication channel. Thus, $j^{\text {th }}$ slave system will receive delayed copies of all $k$-operators forces $\left(F_{m j d}^{i}, i=1,2, \ldots, k\right)$.

After transmitting the composite variables and force signals over the channel, control gains are introduced in line with the composite state convergence scheme. First, $j^{\text {th }}$ slave system is stabilized with $k_{s}^{j}\left(k_{s}^{j}, j=1,2, \ldots, l\right)$. Since, $j^{\text {th }}$ slave system also receives delayed composite variables from all the master systems, gains $r_{s j}^{i}\left(r_{s j}^{i}, i=1,2, \ldots, k\right)$ are introduced to scale the incoming composite variables from the master systems. In addition, operators' forces are also scaled at $j^{\text {th }}$ slave system with $G_{s j}^{i}\left(G_{s j}^{i}, i=1,2, \ldots, k\right)$. The control gains for the master systems are introduced in the same manner. First, $j^{\text {th }}$ master system is stabilized with $k_{m}^{j}\left(k_{m}^{j}, j=1,2, \ldots, k\right)$. Since, $j^{\text {th }}$ master system also receives delayed composite variables from all the slave systems, gains $r_{m j}^{i}\left(r_{m j}^{i}, i=1,2, \ldots, l\right)$ are introduced to scale the incoming composite variables. These $r_{m j}^{i}$ gains are pre-computed as $r_{m j}^{i}=k_{f j}^{i} k_{e}^{i}$ where $k_{f j}^{i}$ is the force-feedback gain from the $i^{\text {th }}$ slave to the $j^{\text {th }}$ master system while $k_{e}^{i}$ is the environment stiffness asSoCiated with the $i^{\text {th }}$ slave system. All other control gains will be determined through the proposed design procedure which is an 

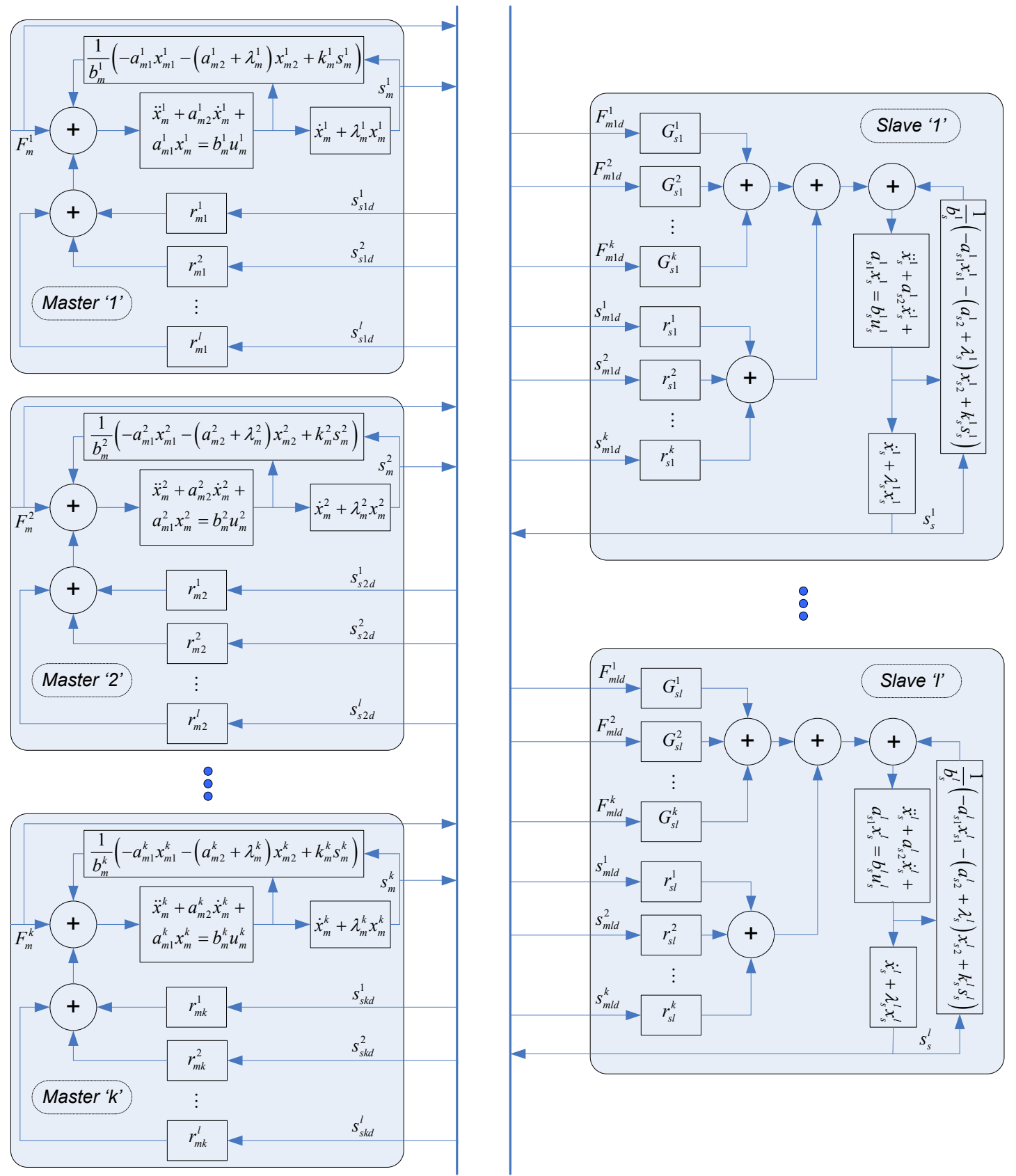

Figure 2. Proposed generalized state convergence scheme for multiple systems

extended version of composite state convergence methodology. The proposed scheme is depicted in Figure 2.

\subsection{Design Procedure}

Lets consider single-degrees-of-freedom master and slave systems as $(z=m, s)$ :

$\dot{x}_{z 1}^{i}=x_{z 2}^{i}$

$\dot{x}_{z 2}^{i}=a_{z 1}^{i} x_{z 1}^{i}+a_{z 2}^{i} x_{z 2}^{i}+b_{z}^{i} u_{z}^{i}$

The composite variables for the master and slave systems are defined as: $s_{z}^{i}=x_{z 2}^{i}+\lambda_{z}^{i} x_{z 1}^{i}$

The control inputs for the master systems are proposed as:

$u_{m}^{1}=\frac{1}{b_{m}^{1}}\left(-a_{m 1}^{1} x_{m 1}^{1}-\left(a_{m 2}^{1}+\lambda_{m}^{1}\right) x_{m 2}^{1}+k_{m}^{1} s_{m}^{1}\right)+\sum_{j=1}^{l} r_{m 1}^{j} s_{s l d}^{j}+F_{m}^{1}$

$u_{m}^{k}=\frac{1}{b_{m}^{k}}\left(-a_{m 1}^{k} x_{m 1}^{k}-\left(a_{m 2}^{k}+\lambda_{m}^{k}\right) x_{m 2}^{k}+k_{m}^{k} s_{m}^{k}\right)+\sum_{j=1}^{l} r_{m k}^{j} s_{s k d}^{j}+F_{m}^{k}$

The control inputs for the slave systems are proposed as: 
$u_{s}^{1}=\frac{1}{b_{s}^{1}}\left(-a_{s 1}^{1} x_{s 1}^{1}-\left(a_{s 2}^{1}+\lambda_{s}^{1}\right) x_{s 2}^{1}+k_{s}^{1} s_{s}^{1}\right)+\sum_{j=1}^{k} r_{s 1}^{j} s_{m 1 d}^{j}+\sum_{j=1}^{k} G_{s 1}^{j} F_{m 1 d}^{j}$

$u_{s}^{l}=\frac{1}{b_{s}^{l}}\left(-a_{s 1}^{l} x_{s 1}^{l}-\left(a_{s 2}^{l}+\lambda_{s}^{l}\right) x_{s 2}^{l}+k_{s}^{l} s_{s}^{l}\right)+\sum_{j=1}^{k} r_{s l}^{j} s_{m l d}^{j}+\sum_{j=1}^{k} G_{s l}^{j} F_{m l d}^{j}$

Using the master control input, the closed-loop composite-master systems can be written as:

$\dot{s}_{m}^{1}=k_{m}^{1} s_{m}^{1}+b_{m}^{1} \sum_{j=1}^{l} r_{m 1}^{j} s_{s}^{j}\left(t-T_{m 1}^{j}\right)+b_{m}^{1} F_{m}^{1}$

$\dot{s}_{m}^{k}=k_{m}^{k} s_{m}^{k}+b_{m}^{k} \sum_{j=1}^{l} r_{m k}^{j} s_{s}^{j}\left(t-T_{m k}^{j}\right)+b_{m}^{k} F_{m}^{k}$

Using the slave control inputs, the closed-loop slave-composite systems can be given as:

$\dot{s}_{s}^{1}=k_{s}^{1} s_{s}^{1}+b_{s}^{1} \sum_{j=1}^{k} r_{s 1}^{j} s_{m}^{j}\left(t-T_{s 1}^{j}\right)+b_{s}^{1} \sum_{j=1}^{k} G_{s 1}^{j} F_{m}^{j}\left(t-T_{s 1}^{j}\right)$

$\dot{s}_{s}^{l}=k_{s}^{l} s_{s}^{l}+b_{s}^{l} \sum_{j=1}^{k} r_{s l}^{j} s_{m}^{j}\left(t-T_{s l}^{j}\right)+b_{s}^{1} \sum_{j=1}^{k} G_{s l}^{j} F_{m}^{j}\left(t-T_{s l}^{j}\right)$

Now, the time delay entities are approximated using first order Taylor expansion as:

$s_{m}^{j}\left(t-T_{s l}^{j}\right) \approx s_{m}^{j}(t)-T_{s l}^{j} \dot{s}_{m}^{j}(t)$

$s_{s}^{j}\left(t-T_{m k}^{j}\right) \approx s_{s}^{j}(t)-T_{m k}^{j} \dot{s}_{s}^{j}(t)$

$F_{m}^{j}\left(t-T_{s l}^{j}\right) \approx F_{m}^{j}-T_{s l}^{j} \dot{F}_{m}^{j}=F_{m}^{j}$

The closed-loop composite-systems under the above approximations can be written as:

$\left[\begin{array}{c}\dot{s}_{m}^{1} \\ \vdots \\ \dot{s}_{m}^{k} \\ \dot{s}_{s}^{1} \\ \vdots \\ \dot{s}_{s}^{l}\end{array}\right]=\left[\begin{array}{cccccc}k_{m}^{1} & \ldots & 0 & b_{m}^{1} r_{m 1}^{1} & \ldots & b_{m}^{1} r_{m 1}^{l} \\ & \ddots & & & \vdots & \\ 0 & \ldots & k_{m}^{k} & b_{m}^{k} r_{m k}^{1} & \ldots & b_{m}^{k} r_{m k}^{l} \\ b_{s}^{1} r_{s 1}^{1} & \ldots & b_{s}^{1} r_{s 1}^{k} & k_{s}^{1} & \ldots & 0 \\ & \vdots & & & \ddots & \\ b_{s}^{l} r_{s l}^{1} & \ldots & b_{s}^{l} r_{s l}^{k} & 0 & \ldots & k_{s}^{l}\end{array}\right]\left[\begin{array}{c}s_{m}^{1} \\ \vdots \\ s_{m}^{k} \\ s_{s}^{1} \\ \vdots \\ s_{s}^{l}\end{array}\right]-$

$\left[\begin{array}{cccccc}0 & \ldots & 0 & b_{m}^{1} T_{m 1}^{1} r_{m 1}^{1} & \ldots & b_{m}^{1} T_{m 1}^{l} r_{m 1}^{l} \\ & \vdots & & & \vdots & \\ 0 & \ldots & 0 & b_{m}^{k} T_{m k}^{1} r_{m k}^{1} & \ldots & b_{m}^{k} T_{m k}^{l} r_{m k}^{l} \\ b_{s}^{1} T_{s 1}^{1} r_{s 1}^{1} & \ldots & b_{s}^{1} T_{s 1}^{k} r_{s 1}^{k} & 0 & \ldots & 0 \\ & \vdots & & & \vdots & \\ b_{s}^{l} T_{s l}^{1} r_{s l}^{1} & \ldots & b_{s}^{l} T_{s l}^{k} r_{s l}^{k} & 0 & \ldots & 0\end{array}\right]\left[\begin{array}{c}\dot{s}_{m}^{1} \\ \vdots \\ \dot{s}_{m}^{k} \\ \dot{s}_{s}^{1} \\ \vdots \\ \dot{s}_{s}^{l}\end{array}\right]+$

$\left[\begin{array}{ccc}b_{m}^{1} & \ldots & 0 \\ & \ddots & \\ 0 & \ldots & b_{m}^{k} \\ b_{s}^{1} G_{s 1}^{1} & \ldots & b_{s}^{1} G_{s 1}^{k} \\ & \vdots & \\ b_{s}^{l} G_{s l}^{1} & \ldots & b_{s}^{l} G_{s l}^{k}\end{array}\right]\left[\begin{array}{c}F_{m}^{1} \\ \vdots \\ F_{m}^{k}\end{array}\right]$

(9)
To write the above expression in a compact form, the following notations are introduced:

$$
\begin{aligned}
& s_{m}=\left[\begin{array}{lll}
s_{m}^{1} & \ldots & s_{m}^{k}
\end{array}\right]^{T}, s_{s}=\left[\begin{array}{lll}
s_{s}^{1} & \ldots & s_{s}^{l}
\end{array}\right]^{T} \\
& B_{m}=\operatorname{diag}\left(b_{m}^{1}, \ldots, b_{m}^{k}\right), B_{s}=\operatorname{diag}\left(b_{s}^{1}, \ldots, b_{s}^{l}\right) \\
& K_{m}=\operatorname{diag}\left(k_{m}^{1}, \ldots, k_{m}^{k}\right), K_{s}=\operatorname{diag}\left(k_{s}^{1}, \ldots, k_{s}^{l}\right) \\
& F_{m}=\left[\begin{array}{lll}
F_{m}^{1} & \ldots & F_{m}^{k}
\end{array}\right]^{T}, G_{s}=\left[\begin{array}{ccc}
G_{s 1}^{1} & \ldots & G_{s 1}^{k} \\
& \vdots & \\
G_{s l}^{1} & \ldots & G_{s l}^{k}
\end{array}\right] \\
& R_{m}=\left[\begin{array}{ccc}
r_{m 1}^{1} & \ldots & r_{m 1}^{l} \\
& \vdots & \\
r_{m k}^{1} & \ldots & r_{m k}^{l}
\end{array}\right], R_{s}=\left[\begin{array}{ccc}
r_{s 1}^{1} & \ldots & r_{s 1}^{k} \\
& \vdots & \\
r_{s l}^{1} & \ldots & r_{s l}^{k}
\end{array}\right] \\
& T_{m}=\left[\begin{array}{ccc}
T_{m 1}^{1} & \ldots & T_{m 1}^{l} \\
& \vdots & \\
T_{m k}^{1} & \ldots & T_{m k}^{l}
\end{array}\right], T_{s}=\left[\begin{array}{ccc}
T_{s 1}^{1} & \ldots & T_{s 1}^{k} \\
& \vdots & \\
T_{s l}^{1} & \ldots & T_{s l}^{k}
\end{array}\right]
\end{aligned}
$$

The closed-loop composite-system in (9) can now be written as:

$$
\begin{aligned}
{\left[\begin{array}{cc}
I_{k} & T_{m} \circ\left(B_{m} R_{m}\right) \\
T_{s} \circ\left(B_{s} R_{s}\right) & I_{l}
\end{array}\right]\left[\begin{array}{c}
\dot{S}_{m} \\
\dot{s}_{s}
\end{array}\right]=} & {\left[\begin{array}{cc}
K_{m} & B_{m} R_{m} \\
B_{s} R_{s} & K_{s}
\end{array}\right]\left[\begin{array}{l}
s_{m} \\
s_{s}
\end{array}\right]+} \\
& {\left[\begin{array}{c}
B_{m} \\
B_{s} G_{s}
\end{array}\right] F_{m} }
\end{aligned}
$$

where '。' denotes the Hadamard product. By letting $D_{m}=T_{m} \circ\left(B_{m} R_{m}\right), D_{s}=T_{s} \circ\left(B_{s} R_{s}\right)$ in above equation and using matrix inversion lemma, the following form can be obtained:

$$
\left[\begin{array}{l}
\dot{s}_{m} \\
\dot{s}_{s}
\end{array}\right]=\left[\begin{array}{ll}
A_{11} & A_{12} \\
A_{21} & A_{22}
\end{array}\right]\left[\begin{array}{l}
s_{m} \\
s_{s}
\end{array}\right]+\left[\begin{array}{l}
B_{1} \\
B_{2}
\end{array}\right] F_{m}
$$

where:

$$
\begin{aligned}
& A_{11}=\left(I_{k}-D_{m} D_{s}\right)^{-1} K_{m}-D_{m}\left(I_{l}-D_{s} D_{m}\right)^{-1} B_{s} R_{s} \\
& A_{12}=\left(I_{k}-D_{m} D_{s}\right)^{-1} B_{m} R_{m}-D_{m}\left(I_{l}-D_{s} D_{m}\right)^{-1} K_{s} \\
& A_{21}=-D_{s}\left(I_{k}-D_{m} D_{s}\right)^{-1} K_{m}-\left(I_{l}-D_{s} D_{m}\right)^{-1} B_{s} R_{s} \\
& A_{22}=-D_{s}\left(I_{k}-D_{m} D_{s}\right)^{-1} B_{m} R_{m}-\left(I_{l}-D_{s} D_{m}\right)^{-1} K_{s} \\
& B_{1}=\left(I_{k}-D_{m} D_{s}\right)^{-1} B_{m}-D_{m}\left(I_{l}-D_{s} D_{m}\right)^{-1} B_{s} G_{s} \\
& B_{2}=-D_{s}\left(I_{k}-D_{m} D_{s}\right)^{-1} B_{m}-\left(I_{l}-D_{s} D_{m}\right)^{-1} B_{s} G_{s}
\end{aligned}
$$

Now, we the following linear transformation is defined:

$\left[\begin{array}{l}S_{m} \\ S_{e}\end{array}\right]=\left[\begin{array}{cc}I_{k} & 0_{k l} \\ -\mathrm{A} & I_{l}\end{array}\right]\left[\begin{array}{l}S_{m} \\ S_{s}\end{array}\right]$ 
where matrix A governs the set-points for the slave systems:

$\mathrm{A}=\left[\begin{array}{ccc}\alpha_{s 1}^{1} & \ldots & \alpha_{s 1}^{k} \\ & \vdots & \\ \alpha_{s l}^{1} & \ldots & \alpha_{s l}^{k}\end{array}\right]$

In addition, $s_{e}=\left[\begin{array}{lll}s_{e}^{1} & \ldots & s_{e}^{l}\end{array}\right]^{T}$ is the compositeerror system with $l$-entries described as:

$s_{e}^{i}=s_{s}^{i}-\sum_{j=1}^{k} \alpha_{s i}^{j} s_{m}^{j}$

The time-derivative of the transformed composite master-error system in conjunction with the earlier composite master-slave system yields:

$\left[\begin{array}{c}\dot{s}_{m} \\ \dot{s}_{e}\end{array}\right]=\left[\begin{array}{cc}\tilde{A}_{11} & \tilde{A}_{12} \\ \tilde{A}_{21} & \tilde{A}_{22}\end{array}\right]\left[\begin{array}{l}s_{m} \\ s_{e}\end{array}\right]+\left[\begin{array}{c}\tilde{B}_{1} \\ \tilde{B}_{2}\end{array}\right] F_{m}$

where:

$$
\begin{aligned}
& \tilde{A}_{11}=A_{11}+A_{12} \mathrm{~A} \\
& \tilde{A}_{12}=A_{12} \\
& \tilde{A}_{21}=\left(A_{21}-\mathrm{A} A_{11}\right)+\left(A_{22}-\mathrm{A} A_{12}\right) \mathrm{A} \\
& \tilde{A}_{22}=A_{22}-\mathrm{A} A_{12} \\
& \tilde{B}_{1}=B_{1} \\
& \tilde{B}_{2}=B_{2}-\mathrm{A} B_{1}
\end{aligned}
$$

As per the guidelines provided by composite state convergence method, the composite-error is allowed to evolve as an autonomous system. This leads to the following $2 \mathrm{kl}$ design conditions:

$\tilde{A}_{21}=0, \tilde{B}_{2}=0$

The remaining $k+l$ design conditions are obtained by assigning the desired dynamic behaviour to the composite master-error system with (19) enforced:

$\left|s I_{k}-\tilde{A}_{11}\right| \times\left|s I_{l}-\tilde{A}_{22}\right|=\left|s I_{k}-P\right| \times\left|s I_{l}-Q\right|$

where $P$ and $Q$ are diagonal matrices with the desired poles for the composite-master and composite-error systems, respectively i.e., $P=\operatorname{diag}\left(p_{1}, \ldots, p_{k}\right), Q=\operatorname{diag}\left(q_{1}, \ldots, q_{l}\right)$.

Now, it is left to show that the slave systems indeed follow the weighted reference motions of the master systems with the proposed algorithm. To this end, observe that composite-error system has a closed loop dynamic of $\dot{s}_{e}+Q s_{e}=0$ which implies that $s_{s}-\mathrm{A} s_{m}=0$ in steady state. Thus, composite-slave systems will attain the weighted reference composite-master states. Since the poles of the composite master systems have also been placed on the left half plane, the composite master states will reach to some final value as determined by the constant operators' forces. This implies that composite slave states will converge which ascertains the stability of composite master and slave systems $\left(\dot{s}_{m}=\dot{s}_{s}=0\right)$. Based on these results, the stability and convergence of the original master and slave systems can be investigated. Let $\lambda_{m}, \lambda_{s}$ be diagonal matrices and $x_{m 1}=\left(x_{m 1}^{1}, \ldots, x_{m 1}^{k}\right)^{T}, x_{m 2}=\left(x_{m 2}^{1}, \ldots, x_{m 2}^{k}\right)^{T}$ $, x_{s 1}=\left(x_{s 1}^{1}, \ldots, x_{s 1}^{l}\right)^{T}, x_{s 2}=\left(x_{s 2}^{1}, \ldots, x_{s 2}^{l}\right)^{T}$. In steady state, $\dot{s}_{z}=\dot{x}_{z 2}+\lambda_{z} x_{z 2}=0(z=m, s)$ implies that $x_{z 2}$ will go to zero. This finding combined with the earlier result $s_{s}-\mathrm{A} s_{m}=0$ yields $x_{s 1}=\lambda_{s}^{-1} \mathrm{~A} \lambda_{m} x_{m 1}$. By selecting the same diagonal entries in $\lambda_{m}, \lambda_{s}$, reference tracking of slave systems is achieved.

Remark 2: The proposed algorithm requires fewer equations, $k+l+2 k l$, to be solved for synchronizing $l$-slaves with the references set by $k$-masters as compared to the extended state convergence architecture (Farooq et al., 2017) which requires the solution of $n \times(k+l)+(n+1) \times k l$ design conditions for achieving the same task.

\section{Simulations Results}

The proposed algorithm is validated in MATLAB Simulink environment by considering two types of teleoperation systems. In the first case, the same numbers of master and salve systems are considered while different numbers of master and slave systems are considered in the second instance. It will be shown that slave systems are able to follow the weighted motion of the master systems and the synchronization is, therefore, achieved.

First, a square teleoperation system is setup in simulations where two masters are communicating with two slaves in the proposed framework. The parameters of the master systems are assumed to be $a_{m 1}^{i}=0, a_{m 2}^{i}=-7.1429, b_{m}^{i}=0.2656$ while slave systems are identified as $a_{s 1}^{i}=0, a_{s 2}^{i}=-6.25, b_{s}^{i}=0.2729$. The time delays in the communication channel are assumed as $T_{m i}^{i}=0.1 s, T_{s i}^{i}=0.3 s,\left.T_{m i}^{j}\right|_{i \neq j}=\left.T_{s i}^{j}\right|_{i \neq j}=0.2 \mathrm{~s}$. It is further assumed that the slaves are interacting with soft environments having stiffness $k_{e}^{i}=20 \mathrm{Nm} / \mathrm{rad}$ and all force feedback gains are considered as $k_{f i}^{j}=0.1$. The alpha factors are selected as $\alpha_{s 1}^{1}=0.7, \alpha_{s 1}^{2}=0.3, \alpha_{s 2}^{1}=0.6, \alpha_{s 2}^{2}=0.4$ while the poles are placed at $p_{1}=-2, p_{2}=-10, q_{1}=-4, q_{2}=-10$.

https://www.sic.ici.ro 
The design conditions are solved using MATLAB symbolic toolbox by discarding the time delays and the following gains are obtained:

$G_{s 1}^{1}=0.6813, G_{s 1}^{2}=0.2920, G_{s 2}^{1}=0.5840, G_{s 2}^{2}=0.3893$

$k_{m}^{1}=-10.6583, k_{m}^{2}=-2.4041, k_{s}^{1}=-3.5162, k_{s}^{2}=-9.4214$

$r_{s 1}^{1}=-15.7894, r_{s 1}^{2}=2.5851, r_{s 2}^{1}=-0.1891, r_{s 2}^{2}=11.6481$

Note that although time delays are ignored in the above calculations, but they are being considered during simulations. This will establish the robustness of the proposed scheme to time delays of the communication channel. Now, the simulations are run with the control gains in (21) and the synchronization results are shown in Figures 3-4. It can be observed that both slaves are following the weighted motion of the master systems. Here the weighted composite references, $s_{s, r e f}^{1}=\alpha_{s 1}^{1} s_{m}^{1}+\alpha_{s 1}^{2} s_{m}^{2}$ and $s_{s, r e f}^{2}=\alpha_{s 2}^{1} s_{m}^{1}+\alpha_{s 2}^{2} s_{m}^{2} \quad$ are defined for the first and second slaves, respectively while the corresponding position references for the slaves are obtained through the proposed algorithm and are being tracked effectively.

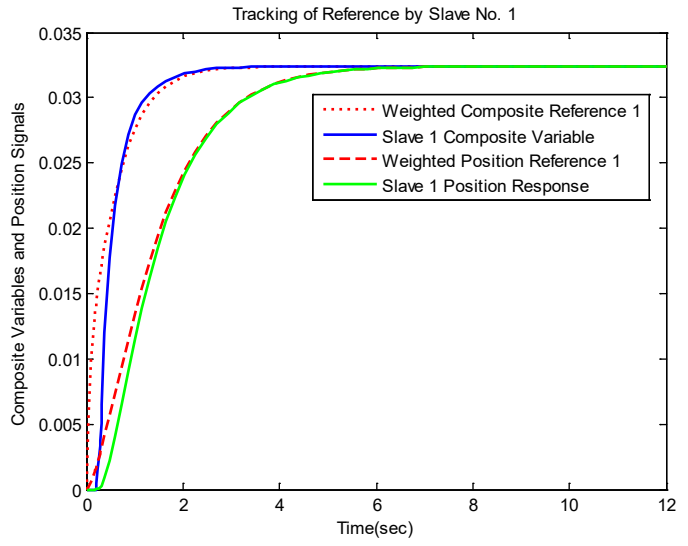

Figure 3. Reference tracking by first slave in $2 \times 2$ teleoperation system

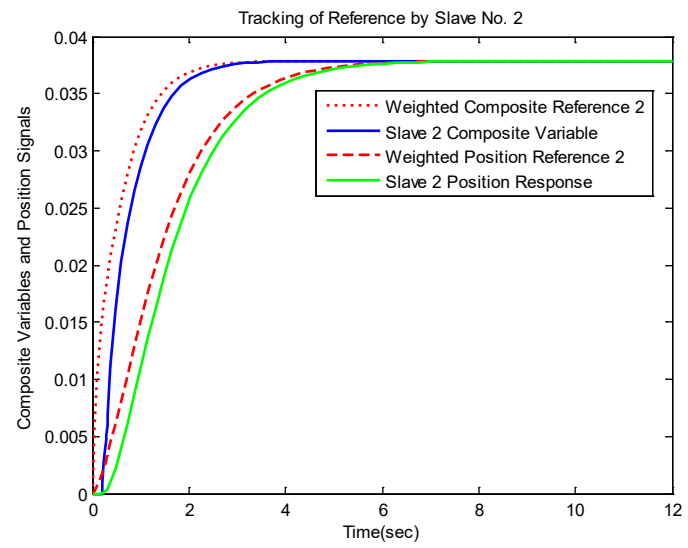

Figure 4. Reference tracking by second slave in $2 \times 2$ teleoperation system
A teleoperation system where three slaves are being operated by a single master is now considered. The parameters for the master and slave systems are the same as those used in the previous example. The poles are placed at $p_{1}=-1.6, q_{1}=-4, q_{2}=-6, q_{3}=-10$. Also, the stiffnesses of the environments are assumed as $k_{e}^{1}=10 \mathrm{Nm} / \mathrm{rad}, k_{e}^{2}=20 \mathrm{Nm} / \mathrm{rad}, k_{e}^{3}=30 \mathrm{Nm} / \mathrm{rad}$. The design conditions are solved with unity alpha factors and ignoring time delay information and the following control gains are obtained:

$G_{s 1}^{1}=G_{s 2}^{1}=G_{s 3}^{1}=0.9733$

$k_{m}^{1}=-3.1936, k_{s}^{1}=-5.7444, k_{s}^{2}=-3.5943, k_{s}^{3}=-9.0677$

$r_{s 1}^{1}=15.1863, r_{s 2}^{1}=7.3080, r_{s 3}^{1}=27.3642$

The teleoperation system is now setup in MATLAB/Simulink environment with the time delays in the communication channel being $T_{m 1}^{1}=T_{s 1}^{1}=0.1 s, T_{m 1}^{2}=T_{s 2}^{1}=0.15 s, T_{m 1}^{3}=T_{s 3}^{1}=0.2 s$

By running the simulations under the control of (22), the results shown in Figure 5 are obtained.

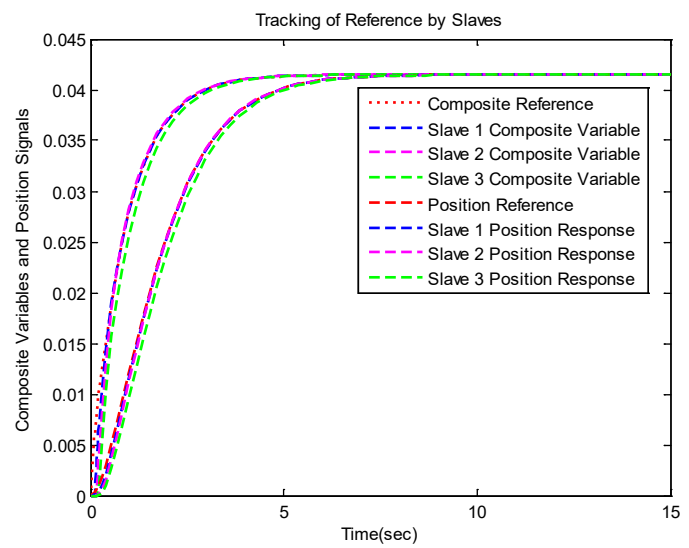

Figure 5. Reference tracking by three slaves in $1 \times 3$ teleoperation system

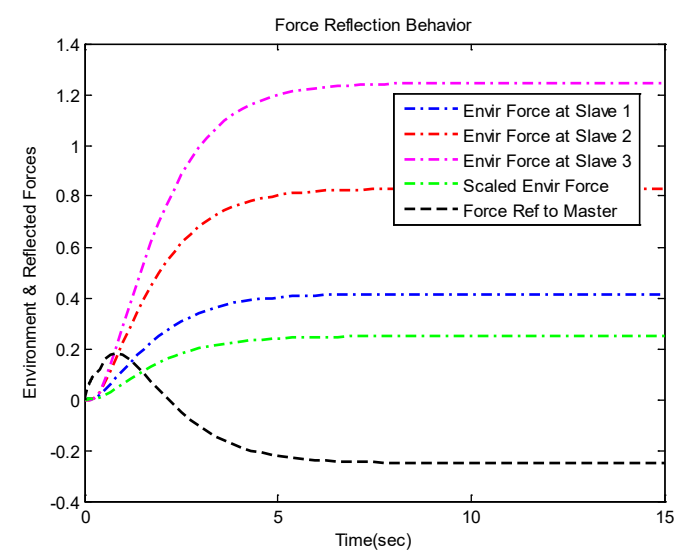

Figure 6. Force reflection behaviour of $1 \times 3$ teleoperation system 
It can be observed that slave systems are following the master system. The force reflection behaviour of $1 \times 3$ teleoperation system is shown in Figure 6. It can be seen the weighted force from slaves, $0.1 \times \sum_{i=1}^{3} F_{e}^{i}$ is reflected to the master in steady state. The above results show that the proposed scheme can indeed accommodate an arbitrary number of master and slave systems. A comparison of proposed scheme with the one presented by Farooq et al. (2017) is shown in Table 1. It can be seen that the proposed scheme requires fewer control gains and communication channels as compared to the extended state convergence architecture while offering a similar performance.

Table 1. Comparison with Farooq et. al (2017)

\begin{tabular}{|c|c|c|c|c|c|}
\hline \multirow{2}{*}{$\begin{array}{l}\text { Sr. } \\
\text { No }\end{array}$} & \multirow{2}{*}{$\begin{array}{l}\text { System } \\
\text { Config. }\end{array}$} & \multicolumn{2}{|c|}{$\begin{array}{l}\text { Number of Control } \\
\text { Gains }(n=2)\end{array}$} & \multicolumn{2}{|c|}{$\begin{array}{l}\text { Number of } \\
\text { Communication } \\
\text { Channels }\end{array}$} \\
\hline & & $\begin{array}{l}\text { (Farooq } \\
\text { et al., } \\
2017 \text { ) }\end{array}$ & Proposed & $\begin{array}{l}\text { (Farooq } \\
\text { et al., } \\
2017 \text { ) }\end{array}$ & Proposed \\
\hline 01 & $2 \times 2$ & 20 & 12 & 20 & 12 \\
\hline 02 & $1 \times 3$ & 17 & 10 & 15 & 9 \\
\hline
\end{tabular}

\section{Experimental Results}

The proposed scheme is also validated through semi-real time experiments. Owing to the availability of a single OMNI device, a $1 \times 3$ teleoperation system is set up, as shown in Figure 7. Only the block connections are shown, as the detailed setup follows Figure 2. The motion of haptic device is constrained to $\mathrm{x}$-axis and an operator's force is generated to drive the master system (2). The master communicates with slaves on the time-delayed channels. The reflected force from the slaves is provided to the haptic device and thus the force feedback loop is closed around the operator. To initiate the experiment, the operator applies a time varying force onto the master by moving the haptic device in the reachable $\mathrm{x}$-direction. The resulting motion of all the slaves is recorded along with the reflected force as sensed by the operator. These data are displayed in Figures 8 and 9. It can be seen that slave systems are tracking the reference motion of the master system while a weighted force is sensed by the operator as well.

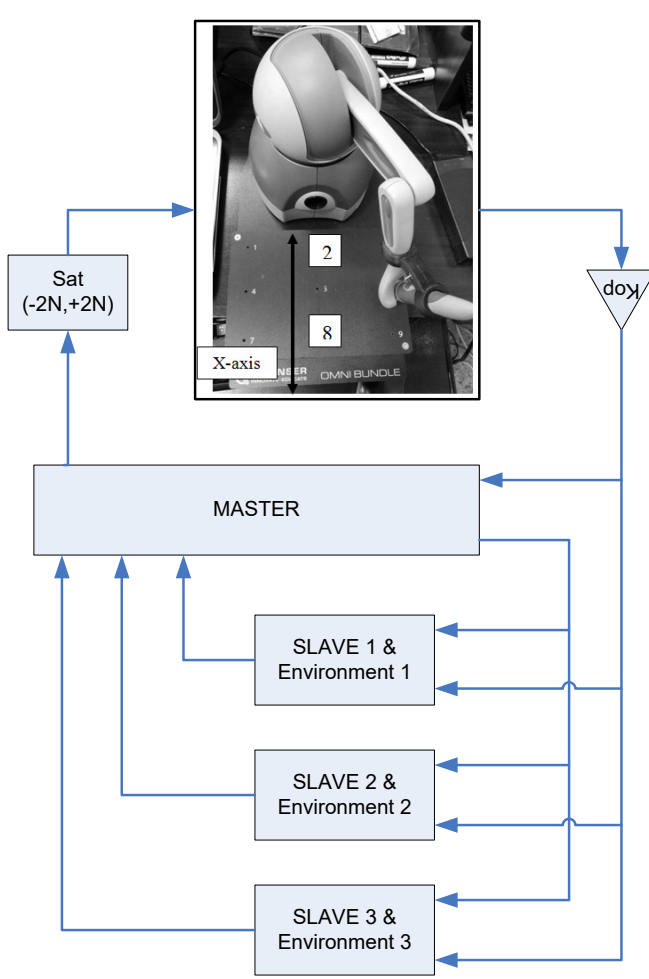

Figure 7. Semi-real time experimental setup for $1 \mathrm{x} 3$ system

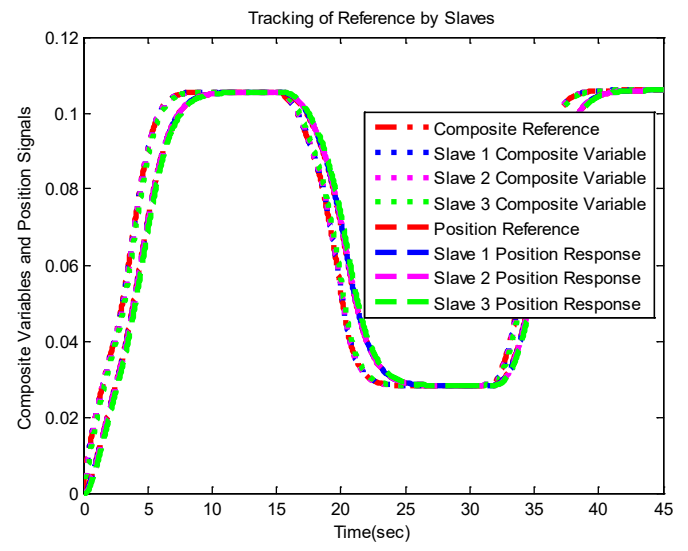

Figure 8. Reference tracking by slaves in $1 \times 3$ teleoperation system of Figure 7

\section{Conclusion}

This paper presents a generalization of composite state convergence scheme with respect to the number of master and slave systems. First, possible interactions between the master and slave systems are considered and the closed loop composite-master and composite-error systems are computed. Second, the composite-error systems are made autonomous and the desired responses are assigned to both the composite-master and composite-error systems which give rise to a total of $\mathrm{k}+1+2 \mathrm{kl}$ design conditions. MATLAB simulations show that the proposed scheme can successfully synchronize 1-slave systems 
with the references set by k-master systems. In future, robustness of the scheme to parametric uncertainties will be analyzed by considering multi-degrees-of-freedom teleoperation systems.

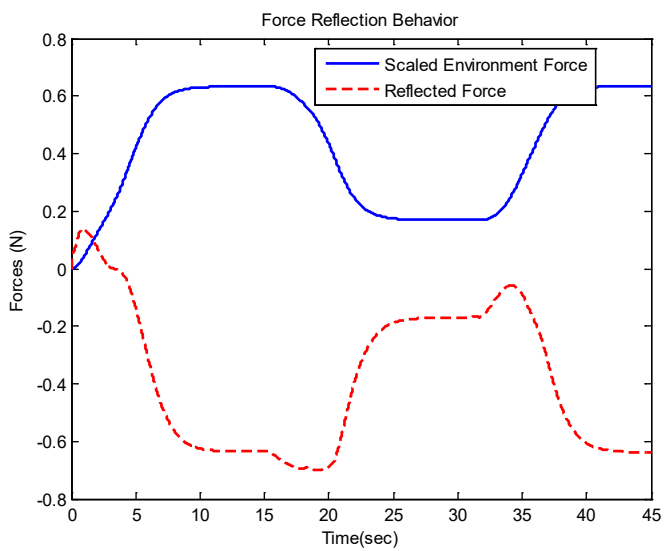

Figure 9. Force reflection behaviour of $1 \times 3$ teleoperation system of Figure 7

\section{Appendix 'A'}

Proposition 1: The closed-loop composite mastererror system of (17) under the control gains found as a solution of (19), (20) is Hurwitz-stable if and only if there exists a symmetric positive definite matrix $P \in R^{(\mathrm{k}+1) \times(\mathrm{k}+1)}$ such that

$$
\begin{aligned}
& {\left[\begin{array}{ll}
P_{11} & P_{12} \\
P_{12}^{T} & P_{22}
\end{array}\right]>0} \\
& {\left[\begin{array}{ll}
\tilde{A}_{11} & \tilde{A}_{12} \\
\tilde{A}_{21} & \tilde{A}_{22}
\end{array}\right]^{T}\left[\begin{array}{ll}
P_{11} & P_{12} \\
P_{12}^{T} & P_{22}
\end{array}\right]+\left[\begin{array}{ll}
P_{11} & P_{12} \\
P_{12}^{T} & P_{22}
\end{array}\right]^{T}\left[\begin{array}{cc}
\tilde{A}_{11} & \tilde{A}_{12} \\
\tilde{A}_{21} & \tilde{A}_{22}
\end{array}\right]<0}
\end{aligned}
$$

The Hurwitz stability of composite mastererror system (17) implies the stability of master-error system as the closed-loop analysis yields $\dot{s}_{z}=\dot{x}_{z 2}+\lambda_{z} x_{z 2}(z=m, s)$. Therefore, the teleoperation system with small constant time delays of the communication channel remains stable as long as (A.1) is satisfied.

The stability of $2 \times 2$ and $1 \times 3$ teleoperation systems from Section 4 will be investigated through the

\section{REFERENCES}

Anderson, R. \& Spong, M. W. (1989). Bilateral control of teleoperators with time delay, IEEE Transactions on Automatic Control, 34(5), 494-501.

Arcara, P. \& Melchiorri, C. (2002). Control schemes for teleoperation with time delay: a comparative study, Robotics and Autonomous Systems, 38(1), 49-64. application of (A.1). By plugging the control gains of (21) in (17) and considering time delays, the system matrix of composite master-error system of $2 \times 2$ teleoperation setup is obtained as:

$$
A=\left[\begin{array}{cccc}
-8.1069 & 0.2066 & 0.7583 & 1.5217 \\
3.0295 & -2.1322 & 0.8564 & 0.8796 \\
-12.9114 & 0.7136 & -3.4446 & 0.5139 \\
-5.0248 & 2.1746 & -1.6064 & -11.5093
\end{array}\right]
$$

The feasibility of Linear Matrix Inequality (LMI) (A.1) yields the following symmetric positive definite matrix which establishes the stability of closed loop teleoperation system:

$P=\left[\begin{array}{cccc}0.3062 & 0.0150 & -0.1533 & 0.0186 \\ 0.0150 & 0.3529 & 0.0575 & 0.0367 \\ -0.1533 & 0.0575 & 0.1693 & -0.0096 \\ 0.0186 & 0.0367 & -0.0096 & 0.0531\end{array}\right]$

Now, let's analyze the stability of $1 \times 3$ teleoperation setup. By substituting (22) and time delay values in (17), the following system matrix is obtained:

$$
A=\left[\begin{array}{cccc}
-1.6160 & 0.5751 & 1.1244 & 3.0831 \\
0.6857 & -6.5578 & -1.5904 & -4.3608 \\
0.4994 & -0.7471 & -5.0551 & -4.0054 \\
2.4295 & -1.4340 & -2.8037 & -16.7554
\end{array}\right]
$$

The solution of LMI (A.1) yields the following symmetric positive definite matrix and hence the stability is verified under small time delays:

$$
P=\left[\begin{array}{cccc}
0.5292 & 0.0215 & 0.0528 & 0.0799 \\
0.0215 & 0.0908 & -0.0119 & -0.0132 \\
0.0528 & -0.0119 & 0.1378 & -0.0182 \\
0.0799 & -0.0132 & -0.0182 & 0.0544
\end{array}\right]
$$

\section{Acknowledgements}

The research reported in this paper has been funded by National Sciences and Engineering Research Council (NSERC) of Canada.

Asad, M., Farooq, U., Gu, J., Abbas, G., Liu, R. \& Balas, V. E. (2019a). A composite state convergence scheme for bilateral teleoperation systems, IEEE/CAA Journal of Automatica Sinica, 6(5),1166-1178.

Asad, M. U., Farooq, U., Gu, J., Balas, V. E., Abbas, G. \& Balas, M. (2019b). Design of a composite state 
convergence controller for a nonlinear telerobotic system, Acta Polytechnica Hungarica, 16(10), 157-172.

Azorin, J. M., Reinoso, O., Aracil, R. \& Ferre, M. (2004). Generalized control method by state convergence of teleoperation systems with time delay, Automatica, 40(9), 1575-1582.

Boukhnifer, M., Chaibet, A., Azib, T. \& Larouci, C. (2012). Design of a tele-control electrical vehicle using a fuzzy logic control, International Journal of Advanced Robotic Systems, 9(1), 1-8.

Chan, L., Naghdy, F. \& Stirling, D. (2014). Application of adaptive controllers in teleoperation systems, IEEE Transactions on Human-Machine Systems, 44(3), 337-352.

Chen, Z., Pan, Y. J. \& Gu, J. (2016). Integrated adaptive robust control for multilateral teleoperation systems under arbitrary time delays, International Journal of Robust and Nonlinear Control, 26(12), 2708-2728.

D'Amore, N. \& Akin, D. L. (2017). Transparency and tuning of wave-based bilateral teleoperation systems, IEEE Robotics and Automation Letters, 2(1), 321-328.

Datta, R., Dey, R. \& Bhattacharya, B. (2019). Further improved stability condition for T-S fuzzy timevarying delay systems via generalized inequality, International Journal of Advanced Intelligence Paradigms, 14(3/4), 310-327.

Del Muro Cuéllar, B., Vazquez Guerra, R. J., Rubio, \& Márquez Rubio, J. F. (2020). Digital two-degreeof-freedom controller for processes with large timedelay, Studies in Informatics and Control, 29(1), 1724. DOI: $10.24846 / v 29 i 1 y 202002$

Farooq, U., Asad, M. U., Gu, J., Abbas, G., Balas, V. E. \& Balas, M. (2018). Design of single master/ multi-slave non-linear teleoperation system through state convergence with time varying delays, Acta Polytechnica Hungarica, 15(8), 55-82.

Farooq, U., Gu, J., El-Hawary, M., Asad, M. U. \& Abbas, G. (2016). Fuzzy model based bilateral control design of nonlinear tele-operation system using method of state convergence, IEEE Access, 4(1), 4119-4135.

Farooq, U., Gu, J., El-Hawary, M., Asad, M., \& Luo, J. (2017). An extended state convergence architecture for multilateral teleoperation systems, IEEE Access, 5(1), 2063-2079.

Ferre. M., Buss, M., Aracil. R, C. Melchiorri, C. \& Balaguer, C. (2007). Advances in Telerobotics. Springer Press.

Hace, A. \& Franc, M. (2013). FPGA implementation of sliding mode control algorithm for scaled bilateral teleoperation, IEEE Transactions on Industrial Electronics, 9(3), 1291-1300.
Hokayem, P. F. \& Spong, M. W. (2006). Bilateral teleoperation: an historical survey, Automatica, 42(1), 2035-2057.

Li, W., Gao, H., Ding, L. \& Tavakoli, M. (2016). Trilateral predictor-mediated teleoperation of a wheeled mobile robot with slippage, IEEE Robotics and Automation Letters, 1(2), 738-745.

Li, Z., Ding, L., Gao, H., Duan, G. \& Su, C. (2013). Trilateral teleoperation of adaptive fuzzy force/ motion control for nonlinear teleoperators with communication random delays, IEEE Transactions on Fuzzy Systems, 2(4), 610-624.

Muradore, R. \& Fiorini, P. (2016). A review of bilateral teleoperation algorithms, Acta Polytechnica Hungarica, 13(1), 191-208.

Niemeyer, G. \& Slotine, J. J. E. (1991). Stable adaptive teleoperation, IEEE Journal of Oceanic Engineering, 16(1), 152-162.

Nozaki, T., Mizoguchi, T. \& Ohnishi, K. (2014). Decoupling strategy for position and force control based on modal space disturbance observer, IEEE Transactions on Industrial Electronics, 61(2), 10221032.

Ryu, J., Kwon, D. \& Hannaford, B. (2004). Stable teleoperation with time-domain passivity control, IEEE Transactions on Robotics and Automation, 20(2), 365-373.

Suzuki, A. \& Ohnishi, K. (2013). Frequency domain damping design for time-delayed bilateral teleoperation system based on modal space analysis, IEEE Transactions on Industrial Electronics, 60(1), 177-190.

Uddin, R. \& Ryu, J. (2016). Predictive control approaches for bilateral teleoperation, Annual Reviews in Control, 42(1), 82-99.

Yan, J. \& Salcudean, S. E. (1996). Teleoperation controller design using $\mathrm{H} \infty$ optimization with application to motion scaling, IEEE Transactions on Control Systems Technology, 4(3), 244-258.

Yang, C., Wang, X., Li, Z., Li, Y. \& Su, C.-Y. (2017). Teleoperation control based on combination of wave variable and neural networks, IEEE Transactions on Systems, Man and Cybernetics, 47(8), 2125-2136.

Yang, X., Hua, C. C., Yan, J. \& Guan, X. P. (2015). A new master-slave torque design for teleoperation system by T-S fuzzy approach, IEEE Transactions on Control Systems Technology, 23(4), 1611-1619.

Zhao, Z., Yang, J., Li, S. \& Chen, W-H. (2019). Composite nonlinear bilateral control for teleoperation systems with external disturbances, IEEE/CAA Journal of Automatica Sinica, 6(5), 1220-1229. 